\title{
A joint latent class model for classifying severely hemorrhaging trauma patients
}

\author{
Mohammad H. Rahbar ${ }^{1,2^{*}}$, Jing Ning ${ }^{3}$, Sangbum Choi ${ }^{1}$, Jin Piao ${ }^{4}$, Chuan Hong ${ }^{4}$, Hanwen Huang ${ }^{5}$,
} Deborah J. del Junco ${ }^{6}$, Erin E. Fox ${ }^{6}$, Elaheh Rahbar ${ }^{7}$ and John B. Holcomb ${ }^{6}$

\begin{abstract}
Background: In trauma research, "massive transfusion" (MT), historically defined as receiving $\geq 10$ units of red blood cells (RBCs) within $24 \mathrm{~h}$ of admission, has been routinely used as a "gold standard" for quantifying bleeding severity. Due to early in-hospital mortality, however, MT is subject to survivor bias and thus a poorly defined criterion to classify bleeding trauma patients.

Methods: Using the data from a retrospective trauma transfusion study, we applied a latent-class (LC) mixture model to identify severely hemorrhaging (SH) patients. Based on the joint distribution of cumulative units of RBCs and binary survival outcome at $24 \mathrm{~h}$ of admission, we applied an expectation-maximization (EM) algorithm to obtain model parameters. Estimated posterior probabilities were used for patients' classification and compared with the MT rule. To evaluate predictive performance of the LC-based classification, we examined the role of six clinical variables as predictors using two separate logistic regression models.

Results: Out of 471 trauma patients, 211 (45 \%) were MT, while our latent SH classifier identified only 127 (27\%) of patients as $\mathrm{SH}$. The agreement between the two classification methods was $73 \%$. A non-ignorable portion of patients (17 out of $68,25 \%$ ) who died within $24 \mathrm{~h}$ were not classified as MT but the SH group included 62 patients (91\%) who died during the same period. Our comparison of the predictive models based on MT and SH revealed significant differences between the coefficients of potential predictors of patients who may be in need of activation of the massive transfusion protocol.
\end{abstract}

Conclusions: The traditional MT classification does not adequately reflect transfusion practices and outcomes during the trauma reception and initial resuscitation phase. Although we have demonstrated that joint latent class modeling could be used to correct for potential bias caused by misclassification of severely bleeding patients, improvement in this approach could be made in the presence of time to event data from prospective studies.

Keywords: Induced censoring, Joint model, Latent variable, Massive transfusion, Mixture, Trauma

\section{Background}

Hemorrhagic shock accounts for the largest proportion of mortality occurring within the first few hours of trauma center care, over $80 \%$ of operating room deaths after major trauma and almost $50 \%$ of deaths in the first $24 \mathrm{~h}$ of trauma treatment [1]. Due to rapidly changing

\footnotetext{
*Correspondence: Mohammad.H.Rahbar@uth.tmc.edu

${ }^{1}$ Division of Clinical and Translational Sciences, Department of Internal

Medicine, The University of Texas Medical School at Houston, The

University of Texas Health Science Center at Houston, Fannin St, Houston,

TX, USA

Full list of author information is available at the end of the article
}

multi-system responses to injury in a relatively shortterm period, highly dynamic treatment regimes with blood transfusion are necessary and make comparative effectiveness research in this area very challenging. In blood transfusion medicine, however, there are no established or universally accepted measures to quantify blood loss or the severity of continuing hemorrhage. To compensate for the lack of quantitative metrics for bleeding severity, a single binary surrogate, namely massive transfusion (MT) stratification, became entrenched in the trauma literature, which is historically defined as the replacement of one's total blood volume by transfusion 
of 10 or more units of red blood cells (RBCs) within $24 \mathrm{~h}$ of admission. This definition has been routinely used to investigate when to initiate a MT protocol, or as a stratification variable to account for potential confounding or effect modification when comparing the effectiveness of different resuscitation protocols [2-8]. However, there is a growing recognition of the pitfalls associated with the use of MT as a surrogate for bleeding severity and the need to replace this poor proxy [9-11]. The shortcomings associated with this classical definition are that it excludes patients who died of hemorrhage-related causes before (1) sufficient numbers of units of blood transfused (e.g., 10th of RBCs) within the specified post-admission time frame (e.g., $24 \mathrm{~h}$ ) to achieve successful resuscitation, and (2) interventions to stop further blood loss (surgical repair of damaged blood vessels and tissue) could be completed.

Several groups have tried to develop better definitions for MT to ameliorate these shortcomings. A recent international forum highlighted twelve different definitions for MT; the most common being $\geq 5$ or 6 RBCs within 4-6 $\mathrm{h}$ [12]. While the time period has been shortened from $24 \mathrm{~h}$, this definition continues to exclude early deaths and does not account for the variability in additional blood products or other hemostatic interventions. An alternative approach has been considering the rate of transfusions. Savage et al. [13] defined "critical administration thresholds" (CAT) of $\geq 3$ units of RBCs per hours to identify hemorrhaging patients. However, the CAT definition is still limited to RBC transfusions and does not account for plasma, platelet transfusions or crystalloids and colloids. More recently, Rahbar et al. [14] reported that 4 units of any resuscitative fluid including blood products, crystalloids and colloids, coined as the "resuscitation intensity", within the first 30 min were predictive of $6 \mathrm{~h}$ mortality in their study. While these definitions are greatly improved from the classical definition of MT, the predictive analysis is still based on simple logistic regressions, which can be viewed as inadequate due to misclassification in the presence of death or informative dropouts [9]. In trauma care, these issues are critical because patient misclassification could result in increased risk of unnecessary blood transfusion or waste of limited and expensive blood resources.

In this article we propose a model-based classification approach for trauma patients. In the past decades, latent class (LC) modeling has been applied in various fields of sciences [15-19]. The goal of LC analysis is to take observed measures (e.g., presence of symptoms or markers of disease) and define a variable that is not directly observable the latent variable (e.g., disease status). These methods have been extended to jointly analyze longitudinal quantitative marker and survival outcome (or informative dropout process), which typically combine a mixed model for longitudinal data and a survival model depending on the latent class [20-24]. Rahbar et al. [11] were the first to apply a LC model to classify patients with severe hemorrhage. This class of models assumes that the dependency between the risk of event and the trajectory of the biomarker is entirely captured by a LC structure rather than by individual random effects. This can avoid many of the numerical complexities of the shared random-effects model under the conditional or so-called 'local' independence assumption. These methods are particularly useful for characterizing heterogeneous populations to more accurately guide clinical decision making.

A unique challenge in analyzing trauma transfusion data is that a terminating or informative censoring event such as death prevents further intervention with blood transfusion. In our example, the total amount of $\mathrm{RBC}$ units transfused prior to death or within $24 \mathrm{~h}$ of admission is dependent upon the duration of a trauma patient's hemodynamically unstable survival. Therefore, the observed blood amount during resuscitation is possibly correlated with patients' survival. Such a dependency, also known as induced censoring, may produce spurious associations and misleading inference if not correctly addressed. To appropriately adjust for a similar induced dependency in medical cost analysis, Lin [25] proposed a linear regression model, accompanied by an inverse probability censoring weighting (IPCW) method. In this article, we consider a LC-based approach that utilizes comprehensive information on patient's presentation, blood usage and survival outcome, with application to a retrospective trauma transfusion study [26]. Specifically, as an alternative to MT classification, using a logistic regression model we introduce a binary latent variable for severe hemorrhage $(\mathrm{SH})$ that classifies severely injured trauma patients who may require massive blood transfusion. The class-specific logistic models for blood product utilization and survival status are then specified under the conditional independence (CI) assumption given each class membership. A benefit of the proposed approach is its ability to incorporate many observable quantities, such as vital signs upon emergency department (ED) admission, into all of these modeling components, as illustrated in Fig. (1), which may better reflect practical complexities and support establishment of a protocol for massive blood transfusion.

Therefore, our goal is to use a LC model to account for induced censoring and correct potential misclassification associated with MT. This research extends the previous work by Rahbar et al. [11] to develop an improved class of LC models that could be used to characterize $\mathrm{SH}$ patients. In addition, we will compare the predictive models developed by the new LC-based classification for 


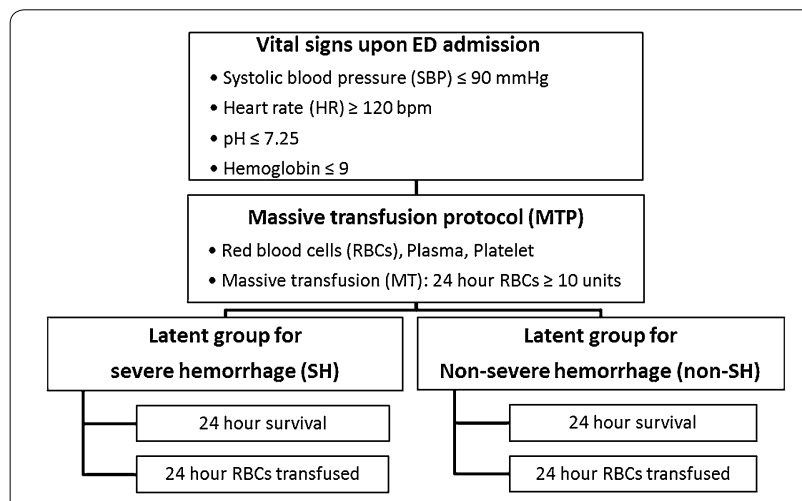

Fig. 1 Diagrams for activation of massive blood transfusion protocol. Actual decision making for massive blood transfusion involves several vital signs at hospital admission and potential risks of blood transfusion and early mortality. The LC-based approach incorporates all these factors while MT is simply based on total utilization of RBCs

$\mathrm{SH}$ with the traditional MT definition. The remainder of this paper is organized as follows. First, the retrospective trauma data are briefly described. The next section describes the statistical model for the biomarker and dropout processes. The performance of our method is evaluated using both simulated data and the data example. A concluding remark is provided in the last section.

\section{The retrospective trauma transfusion study}

This work was motivated by data from a retrospective multi-center trauma transfusion study, which enrolled transfused trauma patients admitted to 16 level 1 trauma centers in the US between July 2005 and June 2006 [26]. Included in the study were 1574 adult trauma patients who arrived from the scene and received at least 1 unit of RBCs in the ED, irrespective of mechanism of injury. Patient characteristics, including age, sex and race, admission vital signs, such as systolic blood pressure $(\mathrm{SBP})$, heart rate (HR), respiratory rate (RR), temperature, hemoglobin (Hgb), and international normalized ratio (INR), Glasgow Coma Scale (GCS), transfusions, admission clinical laboratory tests, prevalence of comorbidity, trips to the operating room and outcome data such as 6- and 24-h mortality and cause of death, were collected from each site and entered into a database at the Department of Epidemiology and Biostatistics, The University of Texas Health Science Center at San Antonio. Given that many patients were intubated upon arrival or in the ED, the respiratory rate was coded as 0 to account for the poor respiratory state. Units of RBCs, platelets, and plasma were adjusted to standard units and totaled at 6 and $24 \mathrm{~h}$ after admission. Crystalloid and colloid amounts were similarly recorded. Ventilator, ICU, and hospital-free days were calculated based on a stay of 30 days. Cause of death was categorized as multiple organ failure, truncal hemorrhage, head injury, airway problems, or others, and validated at each site.

For the analysis, among 1574 patients, 471 with full observations on SBP, HR, Hgb, and $\mathrm{pH}$ were included. Main characteristics (in total and MT vs. non-MT) were summarized in Table 1. The median age was 36 (first and third quartiles $25-52.5)$ years, and 350 patients (74.3\%) were male. Based on the conventional definition of MT,

Table 1 Summary characteristics of trauma patients in the retrospective study

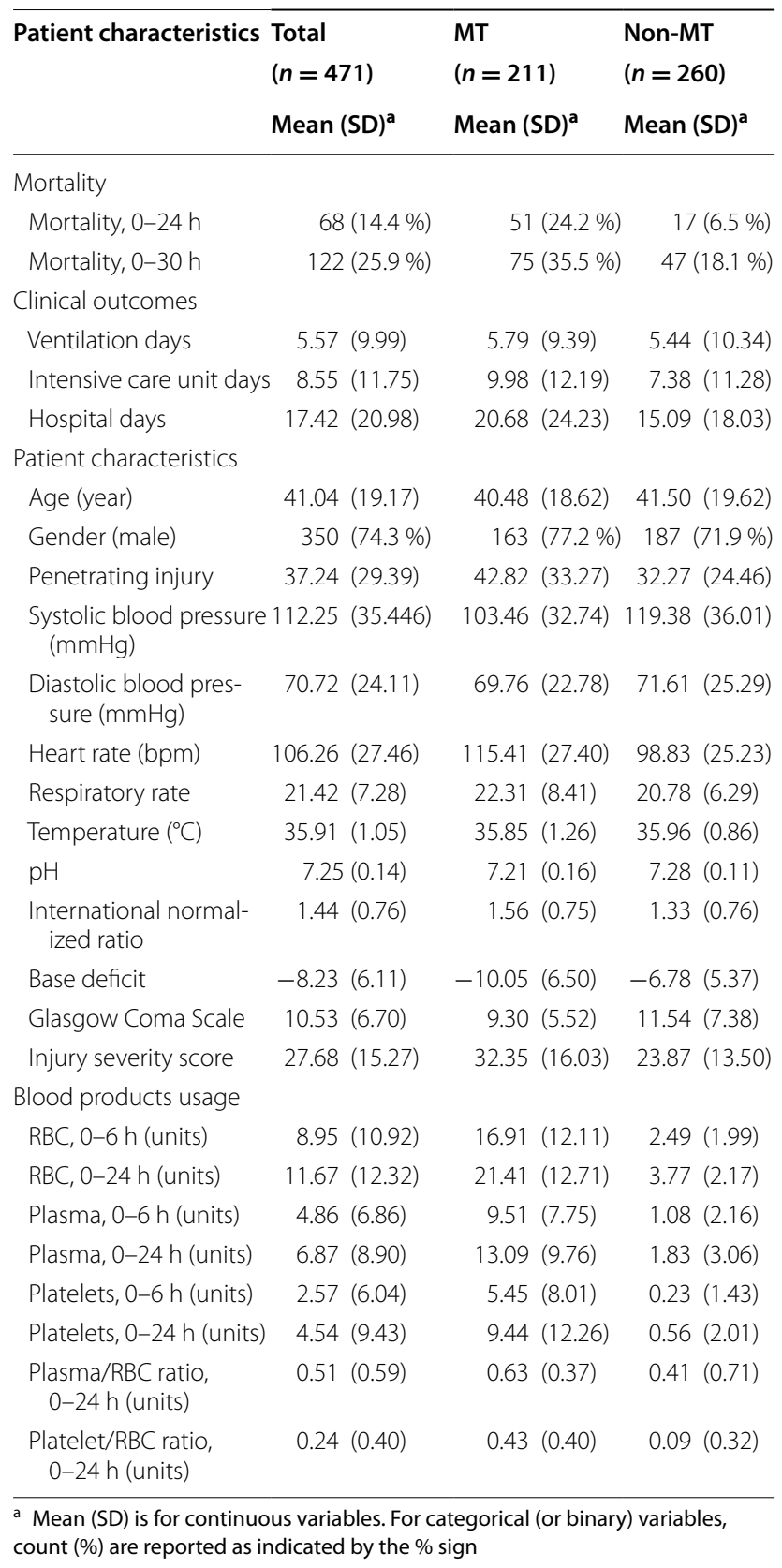


211 (44.8\%) were MT and 260 (55.2 \%) were non-MT. Some patient characteristics, such as base deficit, injury severity score and blood products usage, were substantially different across MT and non-MT. Out of all 471 patients, $68(14.4 \%)$ died in $24 \mathrm{~h}$ and $122(25.9 \%)$ died in $30 \mathrm{~h}$. Among those who died within 24 and $30 \mathrm{~h}$, there were 17 (25.0\%) and 47 (38.5\%) non-MT patients, respectively

\section{Methods}

\section{Model and notation}

We assume that there are two latent homogeneous subgroups and label this latent variable as $\mathrm{SH}$ versus non- $\mathrm{SH}$, where $\mathrm{SH}$ patients are more likely to require activation of a MT protocol. From a statistical perspective, the methodology can be easily generalized to problems with more than two latent classes. Suppose that we have a random sample of $n$ patients. For patient $i \in\{1, \ldots, n\}$, let $g_{i}=\left(g_{i 1}, g_{i 2}\right)$, where $g_{i k}$ is an indicator of membership of class $k=1,2$, and suppose that we observe the biomarker readings $y_{i}$ and the survival indicator $w_{i}$ at $24 \mathrm{~h}$ of hospital admission. By conditional independence, it is assumed that $y_{i}$ and $w_{i}$ are independent given the membership $g_{i}$. The baseline covariates or treatment information will be incorporated into $v_{i}$ for the membership model or $x_{i}$ for the class-specific models. Denoting the conditional distribution of $A$ given $B$ as $[A \mid B]$ and the entire set of parameters by $\Psi$, the log-likelihood can be decomposed as

$l(\Psi)=\sum_{i=1}^{n} \log \left(\sum_{k=1}^{2}\left[g_{i k}=1 \mid v_{i}\right]\left[y_{i} \mid x_{i}, g_{i k}=1\right]\left[w_{i} \mid x_{i}, g_{i k}=1\right]\right)$.

The proposed model can be further described as follows. The probability $\pi_{i 1}=1-\pi_{i 2}$ that subject $i$ belongs to class 1 can be modeled as a function of a vector of covariates $v_{i}$ in a logistic regression with

$$
\pi_{i 1}\left(v_{i}\right)=P\left(g_{i 1}=1 \mid v_{i}\right)=\frac{\exp \left(v_{i}^{T} \alpha\right)}{1+\exp \left(v_{i}^{T} \alpha\right)},
$$

where $\alpha$ is the vector of regression parameters. Next, we assume that the probability of death for class $k=1,2$, depends on the covariates $x_{i}$ through a binary logistic regression:

$$
P\left(w_{i}=1 \mid g_{i k}=1, x_{i}\right)=\frac{\exp \left(x_{i}^{T} \gamma_{k}\right)}{1+\exp \left(x_{i}^{T} \gamma_{k}\right)},
$$

where $\gamma_{k}$ is the $k$ th class-specific coefficient for $k=1,2$. Here, $w_{i}=1$ corresponds to death within $24 \mathrm{~h}, 0$ otherwise. Finally, suppose the response variable $y_{i}$ depends on $x_{i}$ through a linear model: given $g_{i k}=1$,

$$
y_{i}=x_{i}^{T} \beta_{k}+\epsilon_{i k}, \quad \epsilon_{i k} \sim N\left(0, \sigma^{2}\right),
$$

where $\beta_{k}$ is a vector of regression coefficients in class $k$. We assume equal variance for each component in order to avoid the unboundedness of the mixture likelihood. In our trauma data, $y_{i}$ represents the logarithm of cumulative amount of RBCs consumed up to $24 \mathrm{~h}$ or time of death, whichever occurs first, and $w_{i}$ is the survivorship status at $24 \mathrm{~h}$ of hospital admission. However, the exact amount of RBCs transfused at $24 \mathrm{~h}$ is observable only when a patient survives at least for $24 \mathrm{~h}$ (i.e., $w_{i}=0$ ), otherwise, it is censored at the time of death or dropout. Such a phenomenon is common with medical cost data, in which some study subjects are not followed for the full duration of interest so their total costs are unknown for the subjects who are censored. To correct the associated selection bias, Lin [25] adapted an inverse probability of censoring weighted (IPCW) technique to a linear model. This method, however, is not applicable to our situation, because full assessment to survival outcomes is limited with the retrospective data. Instead, we assume that

$$
\exp \left(y_{i}^{\text {obs }}\right) \sim \operatorname{Uniform}\left[0, \exp \left(y_{i}\right)\right], \quad \text { if } \quad w_{i}=1,
$$

that is, the observed amount of RBCs transfused $\left(y_{i}^{\text {obs }}\right)$ is uniformly distributed with true amount $y_{i}$ as the upper boundary. Through a simulation study, we examine the effect of a biased estimation in which censored observations are not adjusted with (4). Although (4) is an untestable assumption, we demonstrated that it is helpful in reducing potential bias caused by induced censoring.

\section{Parameter estimation}

Estimation of the unknown parameters in the proposed mixture model can be performed using a maximum likelihood method. Based on the observed data $\mathbf{O}=\left\{\left(y_{i}^{\text {obs }}, w_{i}, v_{i}, x_{i}\right) ; i=1, \ldots, n\right\}$, the observed likelihood function for $\Psi=\left\{\left(\alpha, \beta_{k}, \gamma_{k}, \sigma\right) ; k=1,2\right\}$ is

$$
\begin{aligned}
& L(\Psi)= \prod_{i=1}^{n}\left[y_{i}, w_{i} \mid x_{i}, v_{i}, \Psi\right] \\
&=\prod_{i=1}^{n}\left(\sum_{k=1}^{2} \pi_{i k}\left(v_{i}\right)\left[y_{i} \mid g_{i k}=1, x_{i}, \Psi\right]\left[w_{i} \mid g_{i k}=1, x_{i}, \Psi\right]\right) \\
&=\prod_{i=1}^{n}\left(\sum _ { k = 1 } ^ { 2 } \frac { \operatorname { e x p } ( g _ { i k } v _ { i } ^ { T } \alpha ) } { 1 + \operatorname { e x p } ( v _ { i } ^ { T } \alpha ) } \left[\left\{\phi\left(\frac{y_{i}^{\mathrm{obs}}-x_{i}^{T} \beta_{k}}{\sigma}\right)\right.\right.\right. \\
&\left.\times\left(\frac{1}{1+\exp \left(x_{i}^{T} \gamma_{k}\right)}\right)\right\}^{I\left(w_{i}=0\right)} \\
& \times\left\{\int_{y_{i}^{\mathrm{obs}}}^{\infty} e^{-\left(u-y_{i}^{\mathrm{obs}}\right)} \phi\left(\frac{u-x_{i}^{T} \beta_{k}}{\sigma}\right)\right. \\
&\left.\left.\left.\times d u\left(\frac{\exp \left(x_{i}^{T} \gamma_{k}\right)}{1+\exp \left(x_{i}^{T} \gamma_{k}\right)}\right)\right\}^{I\left(w_{i}=1\right)}\right]\right),
\end{aligned}
$$

where $\phi(\cdot)$ is a standard normal density. The third equality in (5) follows from conditional independence assumption between $y_{i}$ and $w_{i}$ given all covariates and the latent variable. 
However, it would be cumbersome to maximize the observed-data log-likelihood (5) analytically due to complexities by the presence of mixing parameters and the non-linearity caused by censored observations. To simplify the estimation procedure, we introduce a random variable $z_{i}$ for unobservable $y_{i}$ for the drop out of patient $i$ by death status. We treat latent variables $g_{i}$ and $z_{i}$ as missing data and invoke the expectation-maximization (EM) algorithm to maximize the $\log$-likelihood. Given $g_{i}$ and $z_{i}$, the complete-data log-likelihood is

$$
\begin{aligned}
l_{c}(\Psi)= & \sum_{i=1}^{n} \sum_{k=1}^{2}\left(v_{i}^{T} \alpha\right) g_{i k}-\sum_{i=1}^{n} \log \left\{1+\exp \left(v_{i}^{T} \alpha\right)\right\} \\
& -\frac{n}{2} \log \sigma^{2} \\
& -\frac{1}{2 \sigma^{2}} \sum_{i=1}^{n} \sum_{k=1}^{2} g_{i k}\left(1-w_{i}\right)\left(y_{i}^{\mathrm{obs}}-x_{i}^{T} \beta_{k}\right)^{2} \\
& -\frac{1}{2 \sigma^{2}} \sum_{i=1}^{n} \sum_{k=1}^{2} g_{i k} w_{i}\left(z_{i}-x_{i}^{T} \beta_{k}\right)^{2} \\
& +\sum_{i=1}^{n} \sum_{k=1}^{2} g_{i k}\left[\left(x_{i}^{T} \gamma_{k}\right) w_{i}-\log \left\{1+\exp \left(x_{i}^{T} \gamma_{k}\right)\right\}\right] .
\end{aligned}
$$

In EM algorithm, we alternate between expectation step (E-step) and maximization step (M-step). In the E-step of the $(s+1)$ th iteration, we evaluate the expectation of the complete-data log-likelihood (6), conditional on the observed data $\mathbf{O}$ and the current parameter estimate, say $\Psi^{(s)}$. This is equivalent to calculating the expected values of all the functions of $g_{i}$ and $z_{i}$ that appear in the complete-data log-likelihood. Let $\tilde{E}(\cdot)$ represent such an expectation and $\tilde{g}_{i k}=\tilde{E}\left[g_{i k} \mid \Psi\right]$. The posterior classmembership probability is then

$$
\begin{aligned}
\tilde{g}_{i k} & =\frac{\left[g_{i k}=1 \mid v_{i}\right]\left[y_{i}^{\mathrm{obs}}, w_{i} \mid x_{i}, v_{i}, g_{i k}=1\right]}{\left[y_{i}^{\mathrm{obs}}, w_{i} \mid x_{i}, v_{i}\right]} \\
& =\frac{\pi_{i k}\left(v_{i}\right)\left[y_{i}^{\mathrm{obs}} \mid x_{i}, g_{i k}=1\right]\left[w_{i} \mid x_{i}, g_{i k}=1\right]}{\sum_{k=1}^{2} \pi_{i k}\left(v_{i}\right)\left[y_{i}^{\mathrm{obs}} \mid x_{i}, g_{i k}=1\right]\left[w_{i} \mid x_{i}, g_{i k}=1\right]} .
\end{aligned}
$$

Based on the assumption (4), the $z_{i}^{\prime}$ s have the following class-specific distribution:

$$
\begin{aligned}
p\left(z_{i} \mid y_{i}^{\mathrm{obs}}, g_{i k}=1, \Psi\right)= & \frac{p_{k}\left(z_{i}, y_{i}^{\mathrm{obs}} \mid \Psi\right)}{\int_{y_{i}^{\mathrm{obs}}}^{\infty} p_{k}\left(u, y_{i}^{\mathrm{obs}} \mid \Psi\right) d u} \\
& =\frac{e^{-\left(z_{i}-y_{i}^{\mathrm{obs}}\right)} \phi\left(\frac{z_{i}-x_{i}^{T} \beta_{k}}{\sigma}\right)}{\int_{y_{i}^{\text {obs }}}^{\infty} e^{-\left(u-y_{i}^{\mathrm{obs}}\right)} \phi\left(\frac{u-x_{i}^{T} \beta_{k}}{\sigma}\right) d u},
\end{aligned}
$$

for which we calculate $\tilde{E}_{k}\left[z_{i}^{r} \mid \Psi\right]=\int_{y_{i}^{\text {obs }}}^{\infty} z_{i}^{r} p\left(z_{i} \mid y_{i}^{\text {obs }}\right.$, $\left.g_{i k}=1, \Psi\right) d z_{i} \quad$ for $\quad r=1,2 \quad$ and $\quad k=1,2$. Let $\mathcal{Q}\left(\Psi ; \Psi^{(s)}\right)=\tilde{E}_{g, z}\left[l_{c}(\Psi) \mid \Psi^{(s)}\right]$ be the expected completedata log-likelihood at the $s$ th step, given by

$$
\begin{aligned}
\mathcal{Q}\left(\Psi ; \Psi^{(s)}\right)= & \sum_{i=1}^{n} \sum_{k=1}^{2}\left(v_{i}^{T} \alpha\right) \tilde{g}_{i k}^{(s)} \\
& -\sum_{i=1}^{n} \log \left\{1+\exp \left(v_{i}^{T} \alpha\right)\right\}-\frac{n}{2} \log \sigma^{2} \\
& -\frac{1}{2 \sigma^{2}} \sum_{i=1}^{n} \sum_{k=1}^{2} \tilde{g}_{i k}^{(s)}\left\{\left(1-w_{i}\right)\left(y_{i}^{\mathrm{obs}}-x_{i}^{T} \beta_{k}\right)^{2}\right. \\
& \left.\left.+w_{i} \tilde{E}_{k}\left[\left(z_{i}-x_{i}^{T} \beta_{k}\right)^{2} \mid \Psi^{(s)}\right)\right]\right\} \\
& +\sum_{i=1}^{n} \sum_{k=1}^{2} \tilde{g}_{i k}^{(s)}\left[\left(x_{i}^{T} \gamma_{k}\right) w_{i}\right. \\
& \left.-\log \left\{1+\exp \left(x_{i}^{T} \gamma_{k}\right)\right\}\right]
\end{aligned}
$$

which is maximized in the M-step with respect to $\Psi$; that is, $\Psi^{(s+1)}=\arg \max _{\Psi} \mathcal{Q}\left(\Psi ; \Psi^{(s)}\right)$.

In our normal-mixture model, updating model parameter $\Psi$ in the $(s+1)$ th step is tantamount to calculating

$$
\begin{aligned}
\alpha^{(s+1)}= & \arg \max _{\alpha} \sum_{i=1}^{n}\left[\left(v_{i}^{T} \alpha\right) \tilde{g}_{i k}^{(s+1)}\right. \\
& \left.-\log \left\{1+\exp \left(v_{i}^{T} \alpha\right)\right\}\right], \\
\beta_{k}^{(s+1)}= & \left(X^{T} W_{k}^{(s+1)} X\right)^{-1} X^{T} W_{k}^{(s+1)} \tilde{y}_{k}^{(s)}, \\
\gamma_{k}^{(s+1)}= & \arg \max _{\gamma_{k}} \sum_{i=1}^{n}\left[\tilde{g}_{i k}^{(s+1)}\left(x_{i}^{T} \gamma_{k}\right)\right. \\
& \left.w_{i}-\tilde{g}_{i k}^{(s+1)} \log \left\{1+\exp \left(x_{i}^{T} \gamma_{k}\right)\right\}\right], \\
\sigma^{2(s+1)}= & \frac{1}{n} \sum_{i=1}^{n} \sum_{k=1}^{2} \tilde{g}_{i k}^{(s+1)}\left\{\left(1-w_{i}\right)\left(y_{i}^{\mathrm{obs}}-x_{i}^{T} \beta_{k}^{(s+1)}\right)^{2}\right. \\
& \left.+w_{i} \tilde{E}_{k}\left[\left(z_{i}-x_{i}^{T} \beta_{k}^{(s+1)}\right)^{2} \mid \Psi^{(s)}\right]\right\},
\end{aligned}
$$

where $X=\left(x_{1}, \ldots, x_{n}\right)^{T}, W_{k}^{(s+1)}$ is an $n \times n$ diagonal matrix with diagonal elements $\left\{\tilde{g}_{i k}^{(s+1)}, i=1, \ldots, n\right\}$, $\tilde{y}_{k}^{(s)}=\left(\tilde{y}_{1 k}^{(s)}, \ldots, \tilde{y}_{n k}^{(s)}\right)^{T}$, where $\tilde{y}_{i k}^{(s)}=y_{i}^{\text {obs }}$ if $w_{i}=0$, otherwise, $\tilde{y}_{i k}^{(s)}=\tilde{E}_{k}\left[z_{i} \mid \Psi^{(s)}\right]$. The EM-based maximum-likelihood algorithm updates $\beta_{k}$ by a weighted least squares estimate in the M-step as $\phi(\cdot)$ is a normal density. The EM algorithm is initiated from an initial value $\Psi^{(0)}$, after which one oscillates between the E-step and M-step until convergence is achieved. In order to avoid local maxima for the examples in this paper, the maximization process was repeated 20 times with random starting values. Thus, the reported estimates represent the maximizer over the 
20 maximizations. The use of multiple starting points is quite standard in application of LC models and not terribly onerous for practical purpose. For the examples in this paper, the algorithm converged fairly quickly, and, for the most part, the global maximum was not hard to find.

\section{Standard error estimation}

We estimate standard errors of the estimated class-conditional model and the mixing parameters, using the empirical observed information matrix under the EM algorithm framework,

$$
I_{c}(\hat{\Psi} ; \mathbf{O})=\sum_{i=1}^{n} S_{c}\left(\mathbf{O}_{i} ; \hat{\Psi}\right) S_{c}\left(\mathbf{O}_{i} ; \hat{\Psi}\right)^{T},
$$

where $S_{c}\left(\mathbf{O}_{i} ; \hat{\Psi}\right)$ represents the $i$ th individual completedata score function with respect to the vector of parameters $\Psi$, evaluated at the maximum likelihood estimate $\hat{\Psi}$. The covariance matrix of the parameter estimates is then approximated by the inverse of the empirical Fisher information (9). The appeal of this approach is that all the terms in (9) are by-products of the M-step and provide a reasonable way to estimate standard errors for all model parameters. Wald's test can then be performed based on the estimated variance-covariance matrix.

\section{Classification}

Once the model is fitted, patients can be classified into one of several latent subgroups. In our data example, latent groups can have substantive meaning, such as a group of SH patients for future MT protocol. Although we focus on a two-mixture model, the proposed methodologies can be easily generalized to problems with $K \geq 2$ latent classes. Patients' membership in various subgroups will be determined based on estimated posterior probabilities. We have that $P\left(g_{i k}=1\right)=\pi_{i k}$, termed prior probability; this class probabilities $\pi_{i k}$ represent the likelihood that $i$ th patient belongs to group $k$ but without using information from characteristics of patients, blood usage and survival status. In contrast, the posterior probability of patient $i$ belonging to the $k$ th group is given by (7). This represents how likely is that the $i$ th patient belongs to group $k$, taking into account the observed response $y_{i}^{\text {obs }}$ as well as the survival status $w_{i}$ of that patient. Using these posterior probabilities, we classify patient $i$ into class $k$ if and only if $\tilde{g}_{i k}=\max _{j}\left\{\tilde{g}_{i j}\right\}$. However, in situations where two or more posterior probabilities are almost equal, classification becomes nearly random, which could result in misclassifications. In general, we can vary the number of latent groups $K$ and explore the sensitivity of the classification to the number of latent classes considered. Also, we may use several cut-off points for posterior probabilities and examine whether the results remain consistent.

\section{Results}

Numerical study

In order to assess performance of LC analysis for identifying subpopulations we conducted a simulation study, in which 1000 data sets were simulated, each containing measurements and covariate information from 250 and 500 patients. Mimicking the retrospective trauma study, the LC variable in the model is assumed to split the patient into two latent subgroups. Component probabilities for the LC mixture model follow the logistic model:

$$
\pi_{i 1}\left(v_{i}\right)=1-\pi_{i 2}\left(v_{i}\right)=\frac{\exp \left(\alpha_{0}+\alpha_{1} v_{i}\right)}{1+\exp \left(\alpha_{0}+\alpha_{1} v_{i}\right)},
$$

which involves one covariate $v_{i} \sim N(0,1)$. We let $\alpha=\left(\alpha_{0}, \alpha_{1}\right)^{T}=(0.5,1)^{T}$ so that approximately $60 \%$ of patients belong to class 1 . For the binary survival status, the logistic regression is based on a binary random variable $x_{i} \sim \operatorname{Bernoulli}(0.5)$ :

$$
P\left(w_{i}=1 \mid g_{i k}=1, x_{i}\right)=\frac{\exp \left(\gamma_{0}^{(k)}+\gamma_{1}^{(k)} x_{i}\right)}{1+\exp \left(\gamma_{0}^{(k)}+\gamma_{1}^{(k)} x_{i}\right)}, \quad k=1,2 .
$$

The parameters in these models, $\gamma^{(k)}=\left(\gamma_{0}^{(k)}, \gamma_{1}^{(k)}\right)^{T}$, differ for both latent classes with $\gamma^{(1)}=(1,-1)^{T}$ and $\gamma^{(2)}=(-1,1)^{T}$, corresponding to mortality rates of 62 and $38 \%$ for class 1 and class 2 , respectively. Finally, logarithm of observed RBCs at $24 \mathrm{~h}$ were generated from the class-specific linear model that allowed censoring: when $g_{i k}=1$,

$$
y_{i}^{\mathrm{obs}}=\beta_{0}^{(k)}+\beta_{1}^{(k)} v_{i}+\beta_{2}^{(k)} x_{i}+\Delta_{i}^{(k)}+\epsilon_{i}, \quad \epsilon_{i} \sim N\left(0, \sigma^{2} \mathbf{l} 0\right)
$$

where

$$
\exp \left(\Delta_{i}^{(k)}\right)= \begin{cases}1, & \text { if } w_{i}=0 \\ \text { Uniform }[0,1], & \text { if } w_{i}=1\end{cases}
$$

That is, true cumulative $\mathrm{RBC}$ units can be measured only when the patient is alive $\left(w_{i}=0\right)$, otherwise, observed values will be lower than or equal to the true measurement but at random. We let $\quad \beta^{(1)}=\left(\beta_{0}^{(1)}, \beta_{1}^{(1)}, \beta_{2}^{(1)}\right)^{T}=(\log (15),-1,1)^{T} \quad$ and $\beta^{(2)}=\left(\beta_{0}^{(2)}, \beta_{1}^{(2)}, \beta_{2}^{(2)}\right)^{T}=(\log (8), 1,-1)^{T}, \quad$ so $\quad$ that patients in class 2 will receive generally smaller amount of cumulative RBC units. We consider three scenarios with $\sigma=0.5,1$ and 2, respectively. In this setting, class 1 may represent the $\mathrm{SH}$ subgroup which requires more blood products transfusion. By contrast, conventional MT definition will identify MT patients by the rule: $\exp \left(y_{i}^{\text {obs }}\right) \geq 10$. 
Table 2 contains the results of our simulation study. We calculated the bias of estimates, the empirical standard error (SSE), the average of estimated standard errors (ASE). Besides comparing the mean estimates and true values of the parameters through the bias, we also reported the mean squared error (MSE) that simultaneously involves bias and precision. Simulation results show that bias seems negligible and SEEs and ASEs match reasonably well for all model parameters in three scenarios. Both bias and standard error become smaller as the sample size grows. For the estimation of $\sigma$, we observed some discrepancy between sample and estimated standard errors, but there is no significant impact on the estimation of other regression parameters of interest.

As true value of $\sigma$ increases, the associated error term in model (10) has large variation and thus two latent subgroups are less separable. This was reflected in the increased magnitude of MSE with large $\sigma$. We also note that the proportions that true latent variable coincides with the MT class were about 66,53 and $38 \%$ for $\sigma=0.5,1,2$, respectively, when $n=500$. On the other hand, the corresponding proportions that the estimated posterior probability from (7) correctly predicts the latent class were about 82,74 , and $62 \%$, implying that the LC-based classification consistently outperforms naïve MT classification.

\section{Application to the data from the retrospective trauma transfusion study}

We illustrate application of the proposed method to the data from the retrospective trauma study [26]. The proposed LC model was applied to identify severely hemorrhaging $(\mathrm{SH})$ patients who might need intensive massive transfusion care, assuming that the trauma patients could be split into two or more latent subgroups. The baseline covariates used in our analysis include the following binary patients' characteristics at admission: (1) systolic blood pressure (SBP) $<90 \mathrm{mmHg}$; (2) heart rate (HR) $\geq 120$ bpm; (3) $\mathrm{pH}<7.25$ and (4) Hemoglobin (Hgb) $<9$. These covariates were selected by exploratory analysis and included in models (1)-(3), respectively. In addition, the 24-h blood products ratio, (5) plasma: $\mathrm{RBC}$ ratio and (6) platelet:RBC ratio, were considered as treatment information in models (2) and (3). These two variables are categorized as (ratio $=0),(0<$ ratio $\leq 1$ ), and (ratio $>1$ ). From the observed data, twe can only observe the total amount of RBCs transfused at $24 \mathrm{~h}$ or up to death, whichever comes first.

The proposed LC model was also fitted for different numbers of classes. The values of BIC as the number of classes varied from 1 to 5 were 1689.6, 1362.7, 1366.8, 1368.4 , and 1402.1 respectively, and the associated numbers of parameters were $25,47,73,99$, and 125 . The one-class model is inferior compared with those with more latent classes. The two-class model has the smallest BIC value and may be the favored approach to the data. Hence, the analysis below was based on a two-mixture model for $\mathrm{SH}$ (class 1) versus non-SH (class 2). The class-membership probability, given SBP, HR, $\mathrm{pH}$ and $\mathrm{Hgb}$, can be calculated through estimated coefficients of the logistic model (1). To predict the log-transformed 24-h cumulative RBC transfusion, we used a class-specific linear model (3) and treated 24-h survivorship as a binary response in class-specific logistic models (2), both based on the cumulative 24-h ratios (plasma:RBC and platelet:RBC ratios). The results of the joint LC analysis with (1)-(3) are summarized in Table 3. For comparison purposes, we also carried out separate analyses of the three component models with conventional MT definition.

Overall, the $\mathrm{SH}$ group is characterized by significantly higher units of RBC transfusion than those of the non$\mathrm{SH}$ group (nearly 3 times higher in logarithmic scale), representing that on average the $\mathrm{SH}$ patients received more than 10 units of RBCs within $24 \mathrm{~h}$. The effects of the plasma: $R B C$ ratio and the platelet: $R B C$ ratio on the cumulative 24-h RBC transfusion and the dropout pattern show a clear difference by latent classification. In the $\mathrm{SH}$ subgroup, the higher ratios of plasma/RBC and platelet/RBC were consumed, the lower dropout (death) rates were obtained. The $\mathrm{SH}$ classification will depend on the magnitude of cut-off for posterior probability (7). Because the LC mixture model considered here only contains two latent groups, we merely need to look at one of the posterior probabilities, e.g., the posterior probability that the patient belongs to class 1 . Based on this, the patients can be classified following the suggested cut-off values in Table 4. If the posterior probability lies between 0.45 and 0.55 , it is uncertain to which group the patient can be classified. Only 9 out of 471 patients in the trauma data are in this situation. For the most patients, 450 (95.5\%), it is more clear into which group they can be classified as their posterior probability is above 0.60 .

When the $\mathrm{SH}$ and MT classifications are applied to the same patients, the observed data can be summarized in Table 5. By regarding $\mathrm{SH}$ as "true" binary bleeding status, sensitivity and specificity are 82.7 and $69.2 \%$, implying the possibility that a non-ignorable proportion of trauma patients unnecessarily received MT intervention. Among 68 patients who died before $24 \mathrm{~h}$, a non-ignorable portion of patients $(17,25 \%)$ were not classified as MT but the SH group included 62 patients $(91 \%)$ who died during the same period. Among 22 patients who were non-MT but classified as $\mathrm{SH}, 14$ died before 24 h post admission, while only 3 out of 106 MT but non-SH patients died. 
Table 2 Summary statistics for the simulation studies for the two-component LC mixture model under different scenarios $(\sigma=0.5,1$, and 2$)$ and two sample sizes $(n=250$ and 500$)$

\begin{tabular}{|c|c|c|c|c|c|c|c|c|c|c|}
\hline \multirow[t]{2}{*}{ Scenario } & \multirow[t]{2}{*}{ Parameter } & \multirow[t]{2}{*}{ True } & \multicolumn{4}{|l|}{$n=250$} & \multicolumn{4}{|l|}{$n=500$} \\
\hline & & & Est & SSE & ASE & MSE & Est & SSE & ASE & MSE \\
\hline \multirow[t]{13}{*}{$\sigma=0.5$} & $\alpha_{0}$ & 0.5 & 0.514 & 0.201 & 0.201 & 0.040 & 0.502 & 0.139 & 0.133 & 0.017 \\
\hline & $\alpha_{1}$ & 1.0 & 1.027 & 0.232 & 0.226 & 0.052 & 1.009 & 0.157 & 0.155 & 0.024 \\
\hline & $\beta_{0}^{(1)}$ & 2.708 & 2.708 & 0.071 & 0.073 & 0.005 & 2.710 & 0.050 & 0.050 & 0.002 \\
\hline & $\beta_{1}^{(1)}$ & -1.0 & -1.002 & 0.055 & 0.054 & 0.003 & -1.002 & 0.038 & 0.037 & 0.001 \\
\hline & $\beta_{2}^{(1)}$ & 1.0 & 0.998 & 0.101 & 0.105 & 0.011 & 0.995 & 0.071 & 0.071 & 0.005 \\
\hline & $\beta_{0}^{(2)}$ & 1.609 & 1.605 & 0.141 & 0.133 & 0.017 & 1.610 & 0.096 & 0.094 & 0.009 \\
\hline & $\beta_{1}^{(2)}$ & 1.0 & 1.001 & 0.094 & 0.090 & 0.008 & 1.001 & 0.062 & 0.062 & 0.004 \\
\hline & $\beta_{2}^{(2)}$ & -1.0 & -1.001 & 0.156 & 0.151 & 0.022 & -0.999 & 0.108 & 0.108 & 0.012 \\
\hline & $\gamma_{0}^{(1)}$ & 1.0 & 1.011 & 0.325 & 0.322 & 0.104 & 1.008 & 0.225 & 0.215 & 0.046 \\
\hline & $\gamma_{1}^{(1)}$ & -1.0 & -1.005 & 0.405 & 0.398 & 0.158 & -1.012 & 0.281 & 0.270 & 0.073 \\
\hline & $\gamma_{0}^{(2)}$ & -1.0 & -1.015 & 0.389 & 0.388 & 0.150 & -1.024 & 0.268 & 0.261 & 0.068 \\
\hline & $\gamma_{1}^{(2)}$ & 1.0 & 1.010 & 0.500 & 0.487 & 0.237 & 1.034 & 0.345 & 0.330 & 0.110 \\
\hline & $\log (\sigma)$ & -0.693 & -0.718 & 0.032 & 0.063 & 0.005 & -0.703 & 0.022 & 0.044 & 0.002 \\
\hline \multirow[t]{13}{*}{$\sigma=1$} & $\alpha_{0}$ & 0.5 & 0.522 & 0.272 & 0.267 & 0.072 & 0.507 & 0.187 & 0.185 & 0.034 \\
\hline & $\alpha_{1}$ & 1.0 & 1.034 & 0.281 & 0.283 & 0.079 & 1.009 & 0.190 & 0.183 & 0.034 \\
\hline & $\beta_{0}^{(1)}$ & 2.708 & 2.709 & 0.149 & 0.153 & 0.023 & 2.706 & 0.104 & 0.104 & 0.011 \\
\hline & $\beta_{1}^{(1)}$ & -1.0 & -1.006 & 0.112 & 0.113 & 0.012 & -0.998 & 0.077 & 0.077 & 0.006 \\
\hline & $\beta_{2}^{(1)}$ & 1.0 & 1.001 & 0.206 & 0.203 & 0.043 & 1.003 & 0.147 & 0.145 & 0.021 \\
\hline & $\beta_{0}^{(2)}$ & 1.609 & 1.577 & 0.276 & 0.277 & 0.078 & 1.601 & 0.188 & 0.194 & 0.037 \\
\hline & $\beta_{1}^{(2)}$ & 1.0 & 0.986 & 0.185 & 0.182 & 0.033 & 1.002 & 0.124 & 0.121 & 0.014 \\
\hline & $\beta_{2}^{(2)}$ & -1.0 & -0.980 & 0.298 & 0.301 & 0.091 & -0.995 & 0.206 & 0.213 & 0.045 \\
\hline & $\gamma_{0}^{(1)}$ & 1.0 & 1.005 & 0.366 & 0.348 & 0.121 & 1.005 & 0.253 & 0.251 & 0.063 \\
\hline & $\gamma_{1}^{(1)}$ & -1.0 & -1.003 & 0.443 & 0.415 & 0.172 & -1.003 & 0.307 & 0.298 & 0.089 \\
\hline & $\gamma_{0}^{(2)}$ & -1.0 & -1.014 & 0.44 & 0.427 & 0.182 & -1.012 & 0.308 & 0.310 & 0.096 \\
\hline & $\gamma_{1}^{(2)}$ & 1.0 & 1.016 & 0.558 & 0.516 & 0.266 & 1.021 & 0.385 & 0.400 & 0.160 \\
\hline & $\log (\sigma)$ & 0.0 & -0.027 & 0.065 & 0.065 & 0.005 & -0.012 & 0.045 & 0.042 & 0.002 \\
\hline \multirow[t]{13}{*}{$\sigma=2$} & $\alpha_{0}$ & 0.5 & 0.568 & 0.505 & 0.538 & 0.294 & 0.524 & 0.372 & 0.388 & 0.151 \\
\hline & $\alpha_{1}$ & 1.0 & 1.108 & 0.406 & 0.431 & 0.198 & 1.046 & 0.278 & 0.289 & 0.085 \\
\hline & $\beta_{0}^{(1)}$ & 2.708 & 2.749 & 0.336 & 0.350 & 0.124 & 2.717 & 0.239 & 0.240 & 0.057 \\
\hline & $\beta_{1}^{(1)}$ & -1.0 & -1.051 & 0.262 & 0.266 & 0.073 & -1.023 & 0.188 & 0.193 & 0.037 \\
\hline & $\beta_{2}^{(1)}$ & 1.0 & 1.020 & 0.436 & 0.450 & 0.203 & 0.996 & 0.311 & 0.322 & 0.104 \\
\hline & $\beta_{0}^{(2)}$ & 1.609 & 1.520 & 0.599 & 0.698 & 0.496 & 1.576 & 0.409 & 0.438 & 0.192 \\
\hline & $\beta_{1}^{(2)}$ & 1.0 & 0.951 & 0.406 & 0.453 & 0.207 & 0.971 & 0.271 & 0.284 & 0.081 \\
\hline & $\beta_{2}^{(2)}$ & -1.0 & -1.036 & 0.596 & 0.615 & 0.380 & -1.008 & 0.418 & 0.422 & 0.178 \\
\hline & $\gamma_{0}^{(1)}$ & 1.0 & 1.001 & 0.483 & 0.458 & 0.209 & 1.017 & 0.345 & 0.313 & 0.098 \\
\hline & $\gamma_{1}^{(1)}$ & -1.0 & -1.014 & 0.564 & 0.532 & 0.284 & -1.023 & 0.397 & 0.359 & 0.129 \\
\hline & $\gamma_{0}^{(2)}$ & -1.0 & -0.955 & 0.565 & 0.533 & 0.286 & -1.002 & 0.404 & 0.395 & 0.156 \\
\hline & $\gamma_{1}^{(2)}$ & 1.0 & 0.962 & 0.686 & 0.652 & 0.427 & 1.004 & 0.484 & 0.476 & 0.227 \\
\hline & $\log (\sigma)$ & 0.693 & 0.652 & 0.130 & 0.067 & 0.006 & 0.674 & 0.092 & 0.047 & 0.003 \\
\hline
\end{tabular}




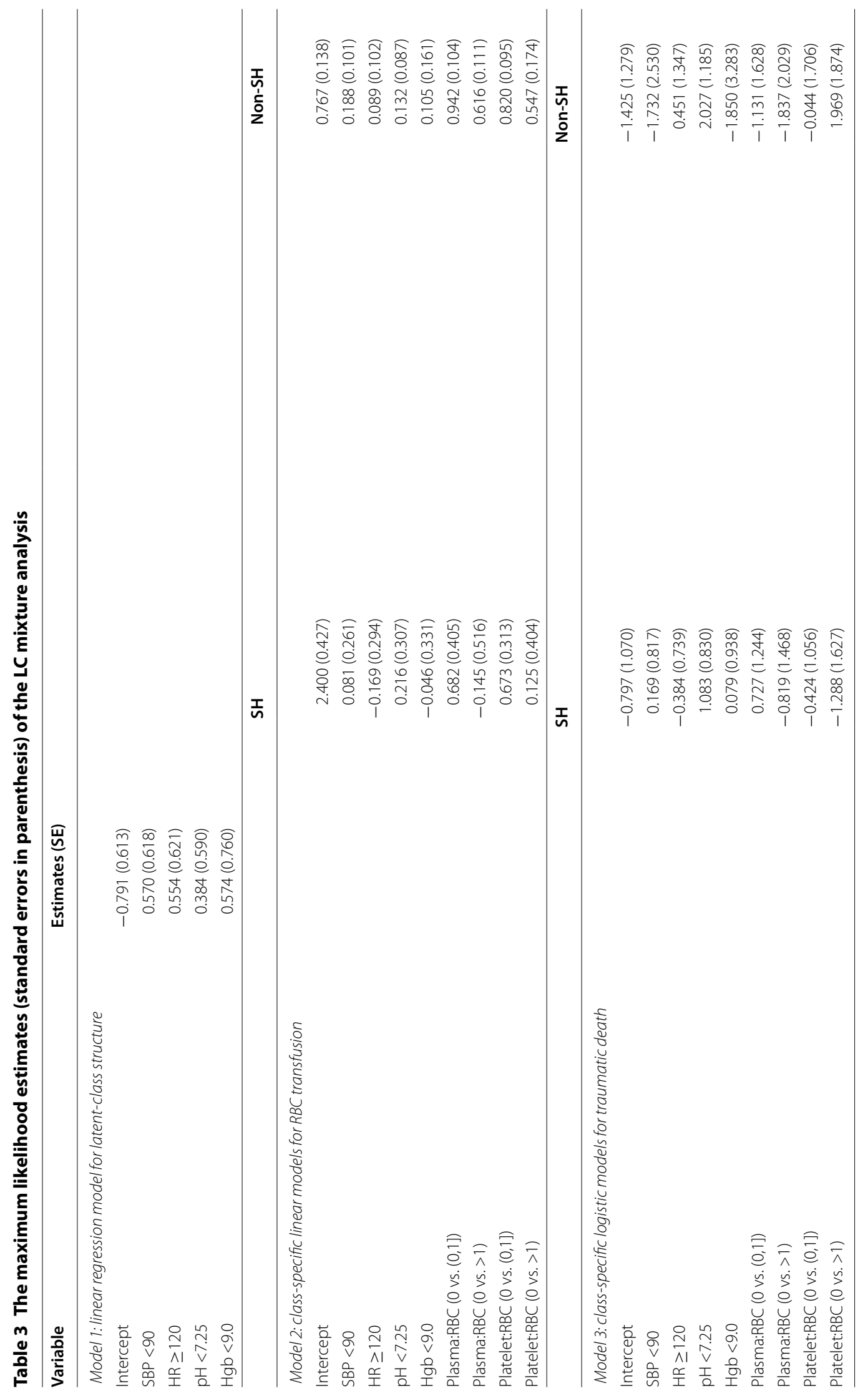


Table 4 Classification of patients based on the posterior probabilities

\begin{tabular}{llc}
\hline Posterior probability & Classification & No. of patients \\
\hline $0.80-1.00$ & Group SH & 83 \\
$0.60-0.80$ & Likely group SH & 34 \\
$0.55-0.60$ & Doubtful, maybe group SH & 6 \\
$0.45-0.55$ & Uncertain & 9 \\
$0.40-0.45$ & Doubtful, maybe group non-SH & 7 \\
$0.20-0.40$ & Likely group non-SH & 61 \\
$0.00-0.20$ & Group non-SH & 271 \\
\hline
\end{tabular}

Table 5 Observed number of patients classified by the LC analysis and conventional MT classification

\begin{tabular}{lccc}
\hline LC analysis & \multicolumn{2}{c}{ Conventional } & Total \\
\cline { 2 - 3 } & Non-MT & MT & \\
\hline Non-SH & 238 & 106 & 344 \\
SH & 22 & 105 & 127 \\
Total & 260 & 211 & 471 \\
\hline
\end{tabular}

Almost half of $\mathrm{SH}$ patients were characterized by early mortality and may be misclassified by the MT definition.

Table 6 presents a summary of comparison between the MT patients who were in the $\mathrm{SH}$ and the non-SH groups. This shows that patients in SH and MT are characterized by higher death rates (46\%) and higher average RBC units transfused (22 units) and relatively lower average blood pressure $(96 \mathrm{mmHg})$ at admission. In contrast, non-SH and MT patients had much lower death rate $(3 \%)$ and consumed fewer blood products than the $\mathrm{SH}$ group. Further comparisons are illustrated in Fig. 2. Patient identification by the observed amount of RBC appears to be less distinct, compared to classification by the posterior probability. Figure 2 further displays the distribution of the predicted RBC units given latent class, by replacing censored observations with their expectations under assumption (4). Clearly, patients in SH had higher RBC transfusions, ranging from 2 to 4 , while RBC units in the non-SH group ranged from 1 to 4 . This also indicates that patients who received a large volume of RBCs may not necessarily belong to the $\mathrm{SH}$ group.

In practice, it is critical to expeditiously identify patients mostly likely to need activation of massive transfusion early in trauma care. Since clinician have been using MT definition as a way to identify early predictors of the need for MT protocol, one could use
Table 6 Summary statistics of 106 non-SH and 105 SH patients both in the MT group

\begin{tabular}{|c|c|c|c|}
\hline & $\begin{array}{l}\text { Non-SH and MT } \\
\text { Mean (SD) }\end{array}$ & $\begin{array}{l}\text { SH and MT } \\
\text { Mean (SD) }\end{array}$ & $P$ value \\
\hline \multicolumn{4}{|l|}{ Patient characteristics } \\
\hline Age (year) & $40.01(17.58)$ & $40.94(19.69)$ & 0.720 \\
\hline Gender (male) & 80 (76 \%) & $83(78 \%)$ & 0.538 \\
\hline $\mathrm{SBP}(\mathrm{mmHg})$ & $110.84(30.79)$ & $96.00(33.09)$ & $0.000^{*}$ \\
\hline $\mathrm{pH}$ & $7.24(0.14)$ & $7.16(0.17)$ & $0.001^{*}$ \\
\hline Heart rate (bpm) & $116.28(27.17)$ & $114.53(27.72)$ & 0.644 \\
\hline Respiratory rate & $21.38(7.83)$ & $23.40(8.97)$ & 0.128 \\
\hline Hemoglobin & $10.76(2.68)$ & $10.80(2.68)$ & 0.915 \\
\hline Death, 0-24 h & $3(3 \%)$ & 48 (46 \%) & $0.000^{*}$ \\
\hline Death, 0-30 h & 19 (18\%) & $56(53 \%)$ & $0.000^{*}$ \\
\hline \multicolumn{4}{|l|}{ Blood product usage } \\
\hline RBC, 0-6 h (units) & $12.08(7.39)$ & $21.79(13.90)$ & $0.000^{*}$ \\
\hline RBC, $0-24 \mathrm{~h}$ (units) & $16.18(8.28)$ & $26.67(14.18)$ & $0.000^{*}$ \\
\hline Plasma, 0-6 h (units) & $7.66(5.92)$ & $11.38(8.87)$ & $0.001^{*}$ \\
\hline Plasma, 0-24 h (units) & $11.20(6.80)$ & $14.98(11.76)$ & $0.005^{*}$ \\
\hline Platelets, 0-6 h (units) & $5.15(7.73)$ & $5.75(8.29)$ & 0.586 \\
\hline Platelets, 0-24 h (units) & $9.21(10.10)$ & $9.65(14.14)$ & 0.795 \\
\hline Plasma/RBC ratio, 0-24 h & $0.71(0.35)$ & $0.55(0.36)$ & $0.001^{*}$ \\
\hline Platelet/RBC ratio, $0-24 \mathrm{~h}$ & $0.48(0.34)$ & $0.37(0.44)$ & $0.037^{*}$ \\
\hline
\end{tabular}

$P$ values were obtained by comparing two subgroups

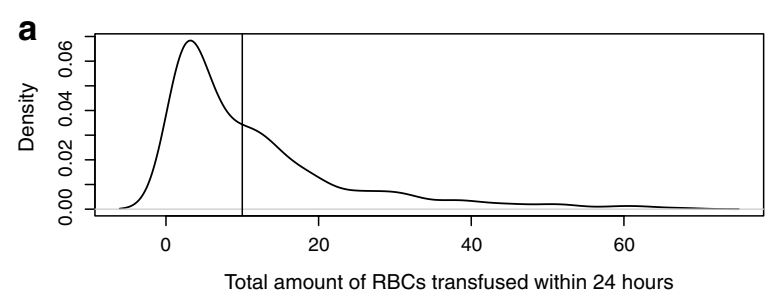

b
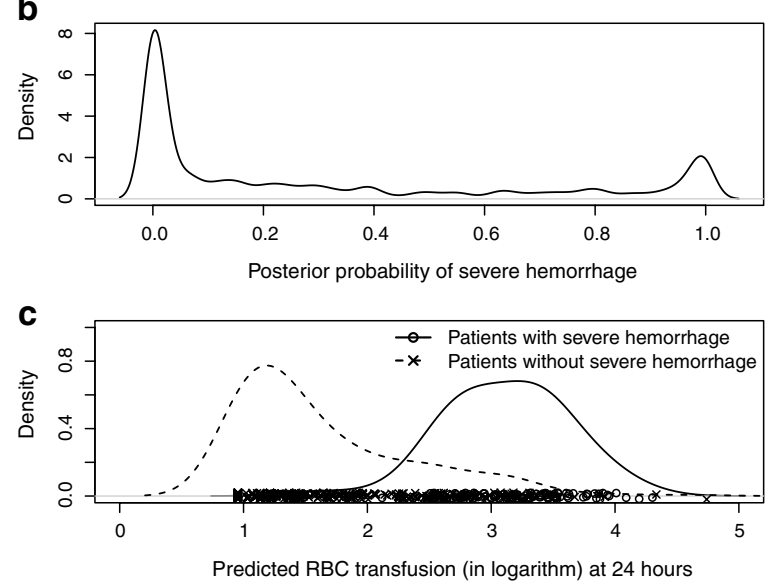

Fig. 2 Distribution of a cumulative amount of RBCs, $\mathbf{b}$ posterior distribution of $\mathrm{SH}$, and $\mathbf{c}$ predicted $\mathrm{RBC}$ units 
the new SH classification for identifying early predictors of SH. It is important to note that for both definitions, MT and $\mathrm{SH}$, one needs to observe patients until hour 24-h. To demonstrate whether prediction models based on MT and SH differ, we performed a multivariable logistic regression using 325 patients and utilizing information from the following variables: SBP of less than $90 \mathrm{mmHg}$, Hgb of less than $11 \mathrm{~g} / \mathrm{dL}$, HR of greater than or equal to $120 \mathrm{bpm}$, temperature of less than $35.5^{\circ} \mathrm{C}$, INR of less than 1.5 , and base deficit (BD) of less than 6. The Wald scores (Table 7) demonstrate the relative weighted influence of each variable, where INR, hemoglobin and heart rate appear to have significant predictability on $\mathrm{SH}$. The predictive equation was $\log [p /(1-p)]=-0.5224+(0.3010 \times \mathrm{SBP})+(0.6628$ $\times \mathrm{HR})+(0.9256 \times \mathrm{Hgb})+(1.6726 \times \mathrm{INR})+(0.1057 \times$ Temperature $)-(0.1648 \times \mathrm{BD})$ with a receivers operating characteristics (ROC) value of 0.73 . The corresponding sensitivity, specificity, positive and negative predictive values are $69,86,38$, and $96 \%$, respectively. We also reported the results from naive analysis, where comparison was made between MT patients and non-MT patients. With respect to percentage of correct decision making, a positive INR (72\%) seems the best individual MT predictor followed by HR (69\%), SBP (68\%), Hgb (63\%). Importantly, all the individual rules remained significant negative predictors (NPV $\geq 75 \%$ ) with SH. Given the clinical utility of the laboratory parameters, particular work may be undertaken to obtain and validate these parameters within the LC framework as we proposed in this paper.

\section{Discussion}

In this study we have used a joint latent class model to improve identification of severely hemorrhaging trauma patients. Because severely bleeding patients may benefit from rapid massive blood transfusion while those with mild blood loss could be potentially harmed by massive blood transfusion, their distinction is critically important but suffers from lack of predictive measurements. Our approach toward this end is to utilize posterior probabilities obtained by the LC method, given information from patient's characteristics and survival information at 24-h post ED admission. The work presented here is considered as an extension of our earlier findings on this topic [11]. The advantage of the proposed method is that it uses admission vital signs to determine the latent variable representing the unknown amount of blood lost (i.e. degree of hemorrhage) in each submodel. Our model-based definition steers away from potential selection biases that could arise when a MT definition depends on a fixed quantity or rate of blood transfusion within a fixed time period. In this study, we found that out of a total of 68 patients who died before $24 \mathrm{~h}, 62(91 \%)$ were identified as $\mathrm{SH}$. The fact that the MT classification misses about $66 \%$ (=91-25\%) of these patients highlighted a major limitation of the classical definition. As a result, the MT definition is not a reasonable surrogate for building predictive models to guide massive blood transfusion protocol.

A number of trauma studies have examined other MT definitions, for example, $\geq 10$ units in $6 \mathrm{~h}$ [2], $\geq 5$ units in $4 \mathrm{~h}$ [7], or assigning patients who died of hemorrhage before receiving 10 units of RBCs into MT as well [27]. Alternatively there have been a few other approaches using rates of transfusions like CAT and 'resuscitation intensity' [13, 14]. However, all of these ad-hoc definitions could under- or over-represent patients who die early, and conversely, may include patients who do not present with critical hemorrhage but develop a need for MT intervention later during the course of their surgical and intensive care phase. Furthermore, it turns out that different MT definitions imply differences in transfusion practices $[7,8,27]$. It should be noted that selection bias from early mortality can be adjusted by using the IPCW technique [25], but such inclusion criteria, solely based on the amount of RBCs, may not fully reflect transfusion practice, which is involved with many other clinical factors, such as usage of other blood products.

Table 7 Predictive models for SH and MT using a multivariate logistic regression

\begin{tabular}{|c|c|c|c|c|c|c|c|c|}
\hline & \multicolumn{4}{|l|}{ SH } & \multicolumn{4}{|l|}{ MT } \\
\hline & Est. & SE & Wald & p value & Est. & SE & Wald & $p$ value \\
\hline (Intercept) & -0.5224 & 0.4348 & -1.20 & 0.2296 & 0.0280 & 0.3910 & 0.07 & 0.9429 \\
\hline $\mathrm{SBP}<90$ & 0.3010 & 0.3422 & 0.88 & 0.3790 & 0.3286 & 0.2895 & 1.14 & 0.2564 \\
\hline$H R \geq 120$ & 0.6628 & 0.3195 & 2.07 & 0.0381 & 0.8856 & 0.2729 & 3.25 & 0.0012 \\
\hline $\mathrm{Hgb}<9.0$ & 0.9256 & 0.4316 & 2.14 & 0.0320 & 0.9077 & 0.4611 & 1.97 & 0.0490 \\
\hline$I N R \geq 1.5$ & 1.6726 & 0.3301 & 5.07 & 0.0000 & 1.1429 & 0.3171 & 3.60 & 0.0003 \\
\hline Temp $<35.5$ & 0.1057 & 0.3597 & 0.29 & 0.7689 & 0.2092 & 0.2809 & 0.74 & 0.4564 \\
\hline $\mathrm{BD}<6$ & -0.1648 & 0.3697 & -0.45 & 0.6558 & 0.0126 & 0.2904 & 0.04 & 0.9653 \\
\hline
\end{tabular}


Using our new SH definition, we have developed predictive models to identify early predictors of the need for MT protocol. Although this definition of SH could be further improved by using time to event data from prospective studies, the purpose of our effort in building predictive models using the definition of $\mathrm{SH}$ is to demonstrate differences in the coefficients of predictive models based on $\mathrm{SH}$ and MT definitions when using the same variables in these predictive models. The data presented in this paper clearly demonstrate a significant difference in the parameter estimates of these predictive models based on the $\mathrm{SH}$ and MT classifications.

It should be noted that this study is limited in being a retrospective review of data on trauma patients entered prospectively, and thus complete information, such as time to death, detailed timing of treatments and blood product utilization was partially available. Consequently, our approach has to rely on a relatively simple parametric model. With full time to event information (e.g., exact time of death), the mortality model in our proposal may be replaced by survival models, such as Cox model. Upon availability of such information, we can also relax the strict 'local' independence assumption, which is likely to be violated in practice. This approach may be applied to a more comprehensive data set from the PRospective Observational Multicenter Major Trauma Transfusion (PROMMTT) study, which is the first large scale, prospective study of trauma patients admitted directly from the injury scene to 10 level-1 trauma centers $[10,28]$. The LC analysis with application to PROMMTT is currently undertaken by our research team, in which we will study broad endpoints of mortality, competing risks and adverse events, such as multisystem organ failure and acute lung injury, etc.

\section{Conclusions}

An accepted definition of MT for trauma resuscitation is vital as it is commonly used to select a study population and drives trauma resuscitation guidelines. The classical MT definition of receiving $\geq 10$ units of RBCs in $24 \mathrm{~h}$ of admission does not adequately reflect transfusion practice and outcome during the ED admission and initial resuscitation phase. Consideration of LC models permits useful joint analysis of biomarker and dropout data and enables bias-corrected estimation of the impact of prognostic features on the main endpoint associated with MT. It also permits full and exact posterior inference for predictive quantity of interest.

\footnotetext{
Abbreviations

BD: base deficit; CAT: critical administration thresholds; Cl: conditional independence; ED: emergency department; EM: expectation-maximization; GCS: glasgow coma scale; Hgb: hemoglobin; HR: heart rate; INR: international normalized ratio; IPCW: inverse probability of censoring weighted; LC: latent class; MT: massive transfusion; MTP: massive transfusion protocol; PROMMTT:
}

PRospective Observational Multicenter Major Trauma Transfusion; RBC: red blood cells; RR: respiratory rate; SBP: systolic blood pressure; $\mathrm{SH}$ : severe hemorrhage.

\section{Authors' contributions}

MHR participated in the design and conduct of the study and writing the manuscript. JN, SC and HH performed the statistical analysis and revised the manuscript. JP and $\mathrm{CH}$ helped the statistical simulation and analysis. DJJ, EF, ER, JBH conceived of the design and coordination of the study and helped revising the manuscript. All authors read and approved the final manuscript.

\section{Author details}

${ }^{1}$ Division of Clinical and Translational Sciences, Department of Internal Medicine, The University of Texas Medical School at Houston, The University of Texas Health Science Center at Houston, Fannin St, Houston, TX, USA. ${ }^{2}$ Division of Epidemiology, Human Genetics and Environmental Sciences, School of Public Health, The University of Texas Health Sciences Center at Houston, Pressler St, Houston, TX, USA. ${ }^{3}$ Department of Biostatistics, The University of Texas MD Anderson Cancer Center, Holcombe Blvd, Houston, TX, USA. ${ }^{4}$ Division of Biostatistics, School of Public Health, The University of Texas Health Sciences Center at Houston, Pressler St, Houston, TX, USA. ${ }^{5}$ Epidemiology and Biostatistics, College of Public Health, University of Georgia, Buck Road, Athens, GA 30602, USA. ${ }^{6}$ Division of Acute Care Surgery, Department of Surgery, Center for Translational Injury Research, The University of Texas Health Science Center at Houston, Fannin St, Houston, TX, USA. ${ }^{7}$ Department of Biomedical Engineering, Wake Forest University, Winston-Salem, NC, USA.

\section{Acknowledgements}

This research is funded by the National Heart, Lung and Blood Institute (NHLBI; R21 HL109479), awarded to The University of Texas Health Science Center at Houston (UTHSC-H). We also acknowledge the support provided by the Biostatistics/Epidemiology/Research Design (BERD) component of the Center for Clinical and Translational Sciences (CCTS) for this project. CCTS is mainly funded by the NIH Centers for Translational Science Award (NIH CTSA) grant (UL1 RR024148), awarded to UTHSC-H in 2006 by the National Center for Research Resources (NCRR) and its renewal (UL1 TR000371) by the National Center for Advancing Translational Sciences (NCATS). The content is solely the responsibility of the authors and does not necessarily represent the official views of the NHLBI or the NCRR or the NCATS.

\section{Competing interests}

The authors declare that they have no competing interests.

Received: 23 October 2014 Accepted: 5 October 2015

Published online: 24 October 2015

\section{References}

1. Kauvar D, Lefering $R$, Wade C. Impact of hemorrhage on trauma outcome: an overivew of epidemiology, clinical presentations, and therapeutic considerations. J Trauma. 2006;60(6 Suppl):S3-11.

2. Kashuk JL, Moore EE, Johnson JL, Haenel J, Wilson M, Moore JB. Postinjury life threatening coagulopaty: is 1:1 fresh frozen plasma:packed red blood cells the answer? J Trauma. 2008;65:261-70.

3. McLaughlin DF, Niles SE, Salinas J, Perkins JG, Cox D, Wade CE, Holcomb JB. A predictive model for massive transfusion in combat casualty patients. J Trauma. 2008;64(S):57-63.

4. Nunez TC, Voskresensky IV, Dossett LA, Shinall R, Dutton WD, Cotton BA. Early prediction of massive transfusion in trauma: simple as abc (assessment of blood consumption)? J Trauma. 2009;66:346-52.

5. Yucel N, Lefering R, Maegele M, Vorweg M, Tjardes T, Ruchholtz S, Neugebauer E, Wappler F, Bouillon B, Rixen D. Trauma associated severe hemorrhage (tash)- score: probability of mass transfusion as surrogate for life threatening hemorrhage after multiple trauma. J Trauma. 2006;60:1228-36.

6. Stanworth SJ, Morris TP, Gaarder C, Goslings JC, Maegele M, Cohen MJ, König TC, Davenport RA, Pittet J-F, Johansson PI, Allard S, Johnson T, Brohi K. Reappraising the concept of massive transfusion in trauma. Crit Care. 2010;14:(R239). 
7. Mitra B, Cameron PA, Gruen RL, Mori A, Fitzgerald M, Street A. The definition of massive transfusion in trauma: a critical variable in examining evidence for resuscitation. Eur J Emerg Med. 2011;18:137-42.

8. Callcut RA, Johannigman JA, Kadon KS, Hanseman DJ, Robinson BR. All massive transfusion criteria are not created equal: defining the predictive value of individual transfusion triggers to better determine who benefits from blood. J Trauma. 2011;70:794-801.

9. del Junco DJ, Fox EE, Camp EA, Rahbar MH, Holcomb JB. Seven deadly sins in trauma outcomes research: an epidemiologic post mortem for major causes of bias. J Trauma Acute Care Surg. 2013;75:97-103.

10. Holcomb JB, del Junco DJ, Fox EE, Wade CE, Cohen MJ, Schreiber MA, Alarcon LH, Bai Y, Brasel KJ, Bulger EM, Cotton BA, Matijevic N, Muskat P, Myers JG, Phelan HA, White CE, Zhang J, Rahbar MH. The prospective, observational, multicenter, major trauma transfusion (PROMMTT) study: comparative effectiveness of a time-varying treatment with competing risks. J Am Med Assoc Surg. 2013;148:127-36.

11. Rahbar $M H$, del Junco DJ, Huang $H$, Ning J, Fox EE, Zhang $X$, Schreiber MA, Brasel KJ, Bulger EM, Wade CE, Cotton BA, Phelan HA, Cohen MJ, Myers JG, Alarcon LH, Muskat P, Holcomb JB. A latent class model for defining severe hemorrhage: experience from the PROMMTT study. J Trauma. 2013;(S82-8).

12. Levi M, Fries D, Gombotz H, van der Linden $P$, Nascimento $B$, Callum JL, Bélisle S, Rizoli S, Hardy JF, Johansson PI, Samama CM, Grottke O, Rossaint R, Henny CP, Goslings JC, Theusinger OM, Spahn DR, Gante MT, Hess JR, Dutton RP, Scalea TM, Levy JH, Spinella PC, Panzer S, Reesink HW. Prevention and treatment of coagulopathy in patients receiving massive transfusions. Vox Sang. 2011;101:154-174.

13. Savage SA, Zarzaur BL, Croce MA, Fabian TC. Redefining massive transfusion when every second counts. J Trauma Acute Care Surg. 2013;74:396-400.

14. Rahbar E, Fox EE, del Junco DJ, Harvin JA, Holcomb JB, Wade CE, Schreiber MA, Rahbar MH, Bulger EM, Phelan HA, Brasel KJ, Alarcon LH, Myers JG, Cohen MJ, Muskat P, Cotton BA. Early resuscitation intensity as a surrogate for bleeding severity and early mortality in the PROMMTT study. J Trauma Acute Care Surg. 2013;75(1 Suppl 1):16-23.

15. Skrondal A, Rabe-Hesketh S. Latent variable modelling: a survey. Scand J Stat. 2007:34:712-45.

16. Garrett ES, Eaton W, Zeger S. Methods for evaluating the performance of diagnostic tests in the absence of a gold standard: a latent class model approach. Stat Med. 2002;21(9):1289-307.

17. Menten J, Boelaert M, Lesaffre E. Bayesian meta-analysis of diagnostic tests allowing for imperfect reference standards. Stat Med. 2013;32:5398-413.
18. Pepe MS, Janes $\mathrm{H}$. Insights into latent class analysis of diagnostic test performance. Biostatistics. 2007;8:474-84.

19. Luo S, Su X, Desantis SM, Huang X, Yi M, Hunt KK. Joint model fora diagnostic test without a gold standard in the presence of a dependent terminal event. Stat Med. 2014; (In Press).

20. Lin H, Turnbull BW, McCulloch CE, Slate EH. Latent class models for joint analysis of longitudinal biomarker and event process data. J Am Stat Assoc. 2002;97:53-65.

21. Proust-Lima C, Letenneur L, Jacqmin-Gadda H. A nonlinear latent class model for joint analysis of multivariate longitudinal data and a binary outcome. Stat Med. 2007:26:2229-45.

22. Beunckens C, Molenberghs $G$, Verbeke $G$, Mallinckrodt C. A latent-class mixture model for incomplete longitudinal Gaussian data. Biometrics. 2008:64:96-105

23. Jacamin-Gadda H, Proust-Lima C, Taylor JM, Commenges D. Score test for conditional independence between longitudinal outcome and time to event given the classes in the joint latent class model. Biometrics. 2010;66:11-9.

24. Proust-Lima C, Séne M, Taylor JM, Jacqmin-Gadda H. Joint latent class models for longitudinal and time-to-event data: a review. Stat Methods Med Res. 2012;23:74-90.

25. Lin DY. Linear regression analysis of censored medical costs. Biostatistics. 2000;1:35-47.

26. Holcomb JB, Wade CE, Michalek JE, Chisholm GB, Zarzabal LA, Schreiber MA, Gonzalez EA, Pomper GJ, Perkins JG, Spinella PC, Kari L, Williams RN, Park MS. Increased plasma and platelet to red blood cell ratios improves outcome in 466 massively transfused civilian trauma patients. Ann Surg. 2008;248:447-56.

27. Callcut RA, Cotton BA, Muskat P, Fox EE, Wade CE, Holcomb JB, Schreiber MA, Rahbar MH, Cohen MJ, Knudson MM, Brasel KJ, Bulger EM, Del Junco DJ, Myers JG, Alarcon LH, Robinson BR. Defining when to initiate massive transfusion: a validation study of individual massive transfusion triggers in PROMMTT patients. J Trauma Acute Care Surg. 2013;74:59-65.

28. Rahbar MH, Fox EE, del Junco DJ, Cotton BA, Podbielski JM, Matijevic N, Cohen MJ, Schreiber MA, Zhang J, Mirhaji P, Duran SJ, Reynolds RJ, Benjamin-Garner R, Holcomb JB. Coordination and management of multicenter clinical studies in trauma: experience from the prospective observational multicenter major trauma transfusion (PROMMTT) study. Resuscitation. 2012;83:459-64.

\section{Submit your next manuscript to BioMed Central and take full advantage of:}

- Convenient online submission

- Thorough peer review

- No space constraints or color figure charges

- Immediate publication on acceptance

- Inclusion in PubMed, CAS, Scopus and Google Scholar

- Research which is freely available for redistribution

Submit your manuscript at

www.biomedcentral.com/submit

C Biomed Central 